(Aus der medizinischen Klinik zu Bonn.)

\title{
Milz und Magenverdauung und der angebliche Pepsingehalt der Milz.
}

$$
\text { Von }
$$

Georg Trampedach (Riga, Livland),

Köln-Kalk, Evangelisches Krankenhaus.

(Mit 2 Textfiguren.)

Die Frage nach der Bedeutung der Milz für den Haushalt des menschlichen Körpers hat praktisches Interesse gewonnen, seitdem die Chirurgen in dringenden Fällen eine planmässige Exstirpation des Organs vorzunehmen wagten. Im Gegensatz zu der Vorstellung von fabelhaften Funktionen, die man der Milz seit altersher zuschrieb, waren die vorgenommenen Exstirpationen von keinen besonderen Ausfallserscheinungen gefolgt. Eine Zusammenstellung derartiger Exstirpationen ist von $K \ddot{\text { chenmeister }}{ }^{1}$ ) sowie von Magdelain ${ }^{2}$ ) und in neuerer Zeit von Brogsitter ${ }^{3}$ ) gemacht worden.

Die ersten Splenektomien im Experiment führte Karl Friedrich Quittenbaum in Rostock an Hunden und Katzen aus, mit dem Resultat, dass die Versuchstiere ohne grobe Ausfallserscheinungen weiterlebten. Weitere Versuche mit dem gleichen Erfolge wurden von Maslowsky ${ }^{4}$, Samuel Wilkes ${ }^{5}$ ) u. a. angestellt.

Eingehende Versuche über die physiologische Tätigkeit der Milz und den Ausfall derselben nach Milzexstirpation führte Moritz Schiff aus. Schiff stützte sich hierbei auf die Beobachtungen von Leuret und Lassaigne, die eine periodische Anschwellung

1) Küchenmeister, Über die wandernde Milz. Leipzig 1865.

2) Magdelain, L'Union p. 146. 1867.

3) Brogsitter, Charité-Annalen Bd. 32 S. 494.

4) Maslowsky, Berliner klin. Wochenschr. Bd. 5 S. 18.1868.

5) Guy's Hosp. Rep. 3. Ser. vol. 11 p. 37. 1865. 
der Milz gesehen hatten, und zwar stets in dem Augenblick, wenn der Chylus in reicher Menge aus dem Magen in den Zwölffingerdarm trat. Aus diesen Beobachtungen, den Arbeiten Corvisart's und seinen eigenen 'Versuchen über die Absonderung des Magenund Pankreassaftes leitete Schiff seine Theorie von einer periodischen Fermentladung des Magens und Pankreas ab.

Nach Schiff schädigt nun die Exstirpation der Milz in erster Hinsicht die Fermentladung des Pankreas. Es tritt keine Ladung ein, da die vom Magen her aus wasserlöslichen Nahrungsmitteln resorbierten Peptogene erst eine Umwandlung in der Milz erfahren müssen, bevor sie im Pankreas abgelagert werden. Da aber nach Schiff's Lehre von der Entstehung des peptischen Magensaftes die Peptogene auch die Fermentladung des Magens bewirken, so müssen nach Milzexstirpation die im Blute kreisenden unverbrauchten Peptogene eine erhöhte Ladung des Magens herbeiführen. Eine Reihe von Versuchen an Hunden und Katzen sehienen diese Theorien zu bestätigen. Das Mageninfus der milzexstirpierten Tiere zeigte höhere Verdauungswerte als das der Kontrolltiere.

Die Verdauungswerte wurden durch die Gewichtsabnahme von Eiweisswürfeln bestimmt, auf die die betreffenden Mageninfuse gewisse Zeit hindurch eingewirkt hatten.

Die Angriffe, die Schiff's Theorien von Heidenhain, A. Fick, Lussana, Grützner und Ebstein ${ }^{3}$ ) erfuhren, richteten sich in erster Linie gegen seine Lebre von der Ladung des Magens durch Peptogene und seine Auffassung von der Pepsinabsonderung.

Schiff's Lehre von der direkten Beeinflussung der Pankreasund indirekten Beeinflussung der Magentätigkeit durch die Vilz fand trotz verschiedener Angriffe in A. Herzen einen eifrigen Verteidiger, der sie in einer Reibe von Publikationen, zum Teil in modifizierter, den neuesten Forschungsergebnissen angemessener Form vertrat.

1) M. Schiff, Gesammelte Beiträge zur Physiologie Bd. 4. Lausanne 1894-1897.

2) Recherches pour servir à l'histoire de la digestion p. 88. Paris 1825. Zitiert nach Schiff.

3) Hermann, Handbuch der Physiologie, und Luciani, Lehrbuch der Physiologie des Menschen. 
Milz und Magenverdauung und der angebliche Pepsingehalt der Milz. 593

Durch die eingehenden Arbeiten von 0 . $\operatorname{Prym}^{1}$ ) ist diese Schiff-Herzen'sche Theorie von den Milz-Pankreasbeziehungen einwandfrei widerlegt worden, was A. Herzen selbst vollständig anerkannte ${ }^{2}$ ). Trotzdem findet sich in Luciani's "Lehrbuch der Physiologie des Menschen" noch die alte Anschauung vertreten. Das mag seinen Grund darin haben, dass der deutschen Übersetzung, Auflage 1906, eine ältere italienische Ausgabe zugrunde liegt.

Mit der Haltlosigkeit der Schiff-Herzen'schen Theorie von der Milz-Pankreasbeeinflussung scheint auch eine Beziehungsannahme zwischen Milz und Magen theoretisch hinfällig geworden zu sein. Trotzdem kann noch eine Reihe von Beobachtungen herangezogen werden, um einen Zusammenhang zwischen diesen beiden Organen wahrscheinlich zu machen. Es ist von verschiedenen Seiten beobachtet worden, dass die zu Versuchszwecken entmilzten Tiere eine grössere Fressgier zeigten. Eine ähnliche Erscheinung berichtet Adelmann ${ }^{3}$ ) in einer Mitteilung über Milzexstirpation an einer 22 jährigen Frau. Die Frau verliess 30 Tage nach der Operation das Spital und befand sich in der Folgezeit dauernd wohl, aber „ihr Appetit war enorm geworden“. Ebenso könnte die starke Gewichtszunahme nach Milzexstirpation bei zwei Patienten, von denen O. Vulpius ${ }^{4}$ ) in seinen „Beiträgen zur Chirurgie und Physiologie der Milz" spricht, in dem Sinne einer Steigerung der peptischen Kraft des Magens gedeutet werden.

In direktem Gegensatz zu dieser Annahme stehen die Ergebnisse der Experimente, die Tarulli und Paskucci in Luciani's ${ }^{5}$ ) Laboratorium (1901) anstellten. Sie fanden die Verdauungskraft des Magensaftes milzexstirpierter Tiere bedeutend herabgesetzt, konnten aber durch Darreichung eines Infuses hyperämisch geschwollener Milz das Verdauungsvermögen des Magens auf $1-3$ Tage wieder steigern. Zum Versuch wurde einem Hunde eine Fistel nach $\mathrm{Cl}$ a u de Bernard angelegt: Vor dem Probefrühstück erhielt er eine vorbereitende Mahlzeit, um das in den Magendrüsen aufgespeicherte Pepsin zu ersehöpfen, Nach 16 Stunden wurde dann die Magen-

1) O. Prym, Pflüger's Arch. 1905.

2) Briefliche Mitteilung an Dr. O. Prym.

3) Deutsche Klinik 1856 Nr. 17.

4) Beiträge zur klin. Chirurgie Bd. 11 S. 94.

5) Luciani, Lehrbuch der Physiologie des Menschen Bd. 2.

Pflüger's Arehiv für Physiologie. Ba. 141. 
schleimhaut mit einer leicht erwärmten, isotonischen Kochsalzlösung abgewaschen.

Zur Bestimmung der Verdauungswerte diente ein Würfel gekochtes Eiweiss $=1 \mathrm{~g}$, der bei $39^{\circ} 24$ Stunden lang in $10 \mathrm{ccm}$ Magensaft liegen blieb. Aus der Gewichtsabnahme des Eiweisswürfels bestimmten Tarulli und Paskucci den Grad der Verdauung.

Luciani erwähnt, dass die Ergebnisse dieser Versuche mit der alten Hypothese Bacelli's (1868) über den Einfluss der Milz auf die Magenverdauung übereinstimmten, führte aber leider für diese Angabe keine Literaturquelle an, so dass wir uns über diese Hypothese nicht haben unterrichten können.

Gross ${ }^{1}$ ) hat die Versuche von Tarulli und Paskucei vermittelst seiner Methode der Verdauungsbestimmung an ein e m milzexstirpierten Patienten der Greifswalder Klinik nachgeprüft. Die Versuche wurden nach Ewald'schem Probefrübstück mit ausgehehertem Mageninhalt ausgeführt. Gross fand bei normalem Salzsäuregehalt die Pepsinverdauung vorübergehend in hohem Grade herabgesetzt. Gleichzeitig bestand eine starke Lymphocytose. Gross schliesst aus dieser einen Beobachtung, „dass nach der Milzexstirpation beim Menschen die Pepsinsekretion fast vollständig versiegt, um ganz langsam und stetig ihre normale Höhe wieder zu erreichen. Gleichzeitig sehen wir das Auftreten einer Lymphocytose, die in demselben Verhältnis, wie die Pepsinsekretion steigt, zur Norm abfällt."

Auf Anregung des Herrn Dr. 0. Prym unternahm ich es, die Versuche von Tarulli und Paskucei und die Beobachtungen von Gross an einigen Versuchstieren nachzuprüfen. Bei dem offenbaren Widerspruch, der zwischen den seinerzeit von Schiff aufgestellten Theorien und deren Widerlegungen einerseits und den Beobachtungen von Tarulli und Paskucei sowie Gross andererseits besteht, erschien mir eine derartige Nachprüfung durchaus angebracht.

Wir stellten vier Serien von Versuchen an:

I. Um uns in dem ersten Versuche möglichst an die von Gross geubte Anordnung zu halten, wurde das dem Versuchshund dargereichte Probefrühstück durch Ansaugen ausgehebert. Wir erhielten Speisebrei, der nicht sauer war, eine Erscheinung, die bei den

1) Zeitschr. f. exper. Pathol. u. Therapie Bd. 8. 1910. 
Milz und Magenverdauung und der angebliche Pepsingehalt der Milz. 595

neueren Anschauungen von der schichtweisen Durchsäuerung des Mageninhalts nicht wundernehmen kann.

Es wurde darauf dem Hunde eine Pawlow'sche Kanüle eingeführt und der nach dem Probefühstück durch dieselbe gewonnene Speisebrei auf seinen Pepsingehalt geprüft. Die Bestimmung wurde durch Nachverdauungsversuche mit Mett'schen Röhrchen, in denen sich durch Hitze koaguliertes Hammelblutserum befand, ausgeführt.

II. Beim zweiten Versuch waren wir bestrebt, die Zufälligkeiten zu vermeiden, die bei einer nicht vollständigen Entleerung des Magens in Versuch I entstehen konnten. Zu diesem Zweck wurde eine den $\mathrm{Pawlow}$ 'schen Kanülen nachgebildete, aber zur leichteren Einführung zerlegbare Kanüle ${ }^{1}$ ) in das Duodenum des Versuchshundes gebracht. Der Durchmesser der Kanüle war recht weit, $2 \mathrm{ccm}, \mathrm{m}$ möglichste Sicherheit zu haben, dass der gesamte Speisebrei sich nach aussen durch die Kanüle entleerte. Einführung vor. Gummiballons, trennenden Scheidewänden und ähnliches vermieden wir absichtlich, um in allen unsern Versuchen genau die gleichen Bedingungen zu haben. Aus dem gleichen Grunde verzichteten wir auch darauf, sauere Flüssigkeit in das Duodenum zu spritzen. Fs kam uns nicht darauf an, die normale Magentätigkeit zu studieren, sondern nur vergleichende Verdaunngswerte zu gewinnen.

Um für das Probefrühstück stets dieselben Bedingungen zu haben, wurden acht Portionen Fleisch, à $100 \mathrm{~g}$, in FrlenmeyerKolben gewogen und sterilisiert. Weiter wurden von getrocknetem, gemahlenem Weissbrot eine gleiche Zahl von Portionen; à $25 \mathrm{~g}$, bergestellt und in Reagenzgläsern gut verschlossen aufbewahrt.

Der gewonnene Speisebrei wurde

1. auf seinen Gehalt an gelöstem Stickstoff im Filtrat geprüft und hierdurch der Grad der Verdauung im Magen festgestellt;

2. mit gleichen Teilen $1 / 10$ normaler Salzsäure versetzt, zu 24 stündiger Nachverdauung aufgestellt und durch Bestimmung des gelösten Stickstoffs im Filtrat der Grad der Nachverdauung ermittelt;

3. dureh Verdauungsproben mit Mett'schen Röhrchen auf seine Verdauungsfähigkeit untersucht.

1) Von O. Prym konstruiert, noch nicht reröffentlicht. 
III. Aus dem Magen eines Versuchshundes wurden an verschiedenen Stellen Schleimhautsticke ausgeschnitten und gleichzeitig die Milz exstirpiert.

Das Infus der gewonnenen Schleimhautstücke untersuchten wir mit Mett'schen Röhrchen auf seinen Pepsingehalt. Nachdem der Hund sich von dem Eingriff vollständig erbolt hatte, wurde er getötet und wieder entsprechende Schleimhautstücke aus entsprechenden Partien des Magens auf ihren Pepsingehalt gepruft.

IV. Einer Anzahl Ratten wurde die Milz exstirpiert. Nachdem sich die Tiere von der Operation erholt hatten, wurden in Zeitabständen eine entmilzte Ratte und ein Kontrolltier getötet und die Infuse der beiden Magen auf ihren relativen Pepsingehalt geprüft.

Sämtliche Operationen sind im Laboratorium der Medizinischen Klinik ausgeführt worden. Herr Dr. O. Prym hatte die Liebenswürdigkeit, mich in die zum Teil recht schwierigen Operationen einzuführen, wofür ich ihm an dieser Stelle meinen verbindlichsten Dank ausspreche.

Nach der obigen Übersicht lassen wir die ausführlichen Versuche folgen:

\section{Versuchsserie I.}

Als Versuchshund dient ein kräftiger junger Foxbastard. Ihm wird am 30. September 1910 eine Pawlow's che Magenkanüle eingeführt.

Operation: Äthermorphiumnarkose. Schnitt in der Linea alba. Der Magen wird vorgezogen. Einschnitt im Fundusteil. Die Kanüle wird durch diese Öffnung eingeführt. Der kugelige Versehlussteil der Kanüle wird im Pylorusteil von innen gegen die Schleimhaut gepresst und diese durch Gegensticheln von aussen zur Perforation gebracht. Dann wird mit einem Troikart an entsprechender Stelle die Bauchwand durchstossen und die Kanüle hindurchgeführt. Eine nach Art der Mutterschrauben aufgeschraubte Platte verhindert ihr Zurückgleiten in die Bauchhöhle. Die Schnittwunden werden durch Naht sorgfältig geschlossen und der Bauchschnitt durch eine Schicht Kollodium vor Infektion geschützt.

Der Hund ist bereits am Tage nach der Operation recht munter, so dass am 2. Oktober 1910 mit den Versuchen begonnen werden kann. Der Hund erhält Probefrühstück, bestehend aus einem halben Brötchen und $100 \mathrm{ccm}$ Wasser. Nach $3 / 4$ Stunden wird die Kanüle geöffnet und das Filtrat des ausfliessenden Speisebreis auf seine Verdauungsfähigkeit geprüft.

Zur Prüfung benutzten wir zuerst die Methode Gross ${ }^{1}$ ), konnten uns aber nicht mit derselben befreunden. Es gelang uns nach Zusatz von Natronlauge und Ansäuern mit Essigsäure nicht, die Kaseinlösung vollkommen klar zu be-

1) Zeitschr. f. exper. Pathol. u. Therapie Bd. 8. 
kommen. Auch bei grösseren Zusätzen Filtrat fand sich stets eine gleichbleibende ganz geringe Trübung. Wir betrachteten daraufhin als Verdauungsgrenze dasienige Gläschen, bei welchem diese gleichbleibende geringe Trübung sich zu steigern begann. Durch Zufall blieb eine erledigte Serie Gläschen 24 Stunden lang stehen, und da zeigte es sich, dass auch einige Gläschen mit. der geringen Trübung flockigen Niederschlag hatten. Wir stellten nun zwei ganz gleich beschickte Serien her und liessen sie nach Behandlung mit Natronlauge und Essigsäure 24 Stunden stehen. Ein Teil der Gläschen enthielt flockigen Niederscblag, und zwar hörte derselbe in beiden Serien nicht in zwei korrespondierenden Gläschen auf, sondern in verschiedenen. Da wir eine Nachprüfung der Methode Gross nicht als unsere Aufgabe betrachten konnten, so gaben wir die Versuche auf und benutzten später stets die alte bewährte Methode der Mett'schen Röhrchen zur Verdauungsbestimmung. Wir wählten diese Methode, trotzdem uns die Einwände bekannt sind, die gegen dieselbe erhoben werden, da es uns nicht auf absolute, sondern nur auf vergleichende Werte der Verdauung ankam.

Bei den späteren Versuchen mit Milzinfus haben wir noch einmal die Gross'sche Methode benutzt, um festzustellen, ob überhaupt eine Verdauung vorhanden war.

Gleichzeitig mit den Verdauungsproben wurde das Blut des Versuchshundes auf seinen Gehalt an weissen Blutkörperchen untersucht und der prozentuale Gehalt an Lymphocyten festgestellt. Das Ergebnis der Verdauungs- und Blutuntersuchungen vor und nach der Milzexstirpation geht aus den nachfolgenden Kurven hervor. Die Verdauungswerte sind durch Mett'sche Röhrchen, die mit Hammelblutserum gefüllt waren, bestimmt und in der Kurve in Milimetern ausgedrückt. Der Aufenthalt der Röhrchen im Brutschrank betrug 24 Stunden bei $37^{\circ}$. Jedesmal wurden Gesamtazidität und Salzsäuregehalt des Probefrühstücks bestimmt. Die ermittelten Werte sind in der Kurve eingezeichnet.

Der Hund äberstand die Entmilzung gut. Sie wurde am 15. November ausgeführt. Am 18. November erhielt er wieder das erste Probefrühstück.

Fig. 1 gibt die Werte der Verdanung, Azidität und des Salzsäuregehaltes an.

Fig. 2 enthält die im 1/100 qmm gefundenen Zahlen der Leukocyten und Lymphocyten.

Aus den obigen Versuchen geht im Gegensatz zu den Beobachtungen von Tarulli und Paskucci und der Beobachtung ron Gross deutlich hervor, dass die Entmilzung keinen verringernden Einfluss auf die peptische Kraft des Magensaftes hatte. Nach der Entmilzung war, wie aus Fig. 1 hervorgeht, kein dauernder Tiefstand der Verdauungswerte $\mathrm{zu}$ verzeichnen. Die Werte schwanken, bewegen sich aber in der Mehrzahl in denselben Grenzen wie vor der Entmilzung. Auch sonst wurden keine pathologischen Erscheinungen an dem Versuchshund beobachtet. Lymphdrüsenschwellung trat nicht ein. Die Änderung des Blutbildes nach der Entmilzung durch starke Zunahme der Lymphocyten stimmt mit den Angaben 
von Gross überein. Dagegen haben wir nicht finden können, dass die Zunahme der Lymphocyten mit einer Herabsetzung der Magentätigkeit parallel ging. Wir beobachteten auch im Gegensatz zu Gross einen Anstieg der Gesamtzahl weisser Blutkörperchen.

Lymphocytose nach Milzexstirpation ist von einer grösseren Zahl Autoren bereits beobachtet worden. So durch die von Gross zitierten Autoren Hartmann und Vaques ${ }^{1}$ ) sowie Hoffmann ${ }^{2}$ ).

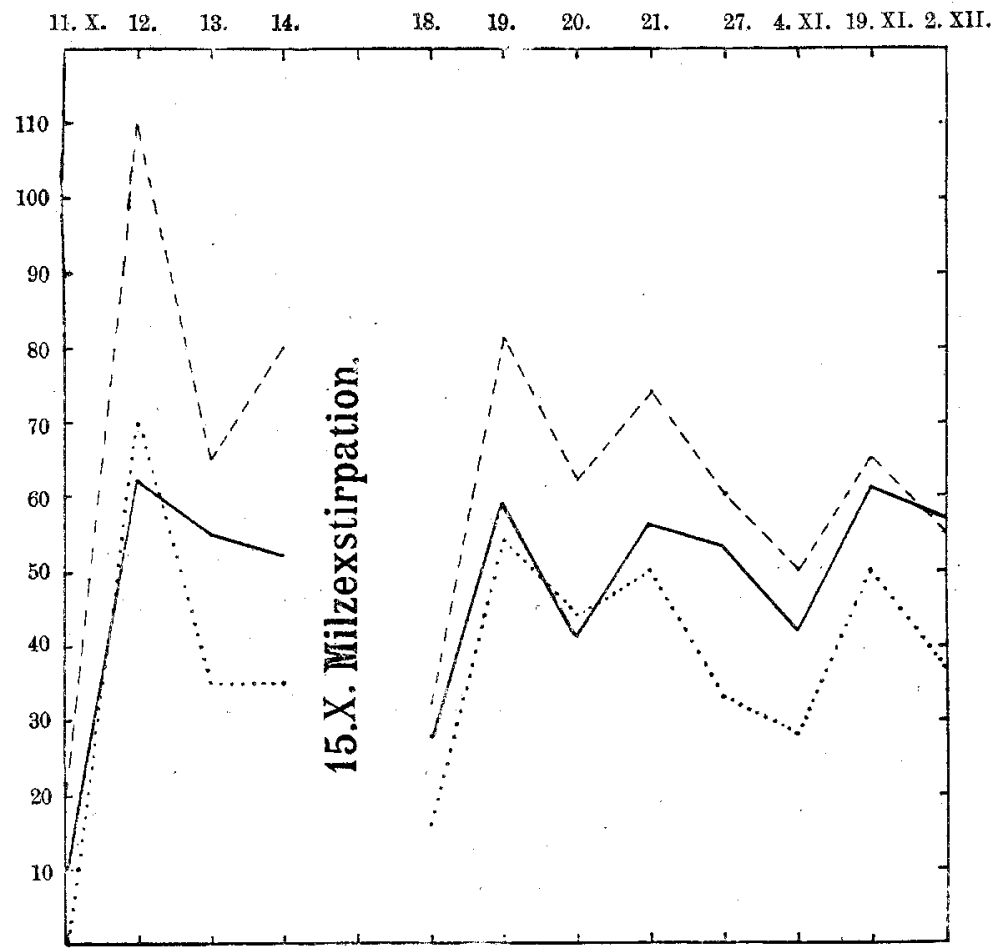

Fig. 1. $-=$ Verdauungswert. $-\cdots=$ Gesamtazidität. $\cdots \cdots .=$ freie Salzsäuren. Die Verdauungswerte sind in Millimetern, multipliziert mit 10, ausgedrückt, Azidität und Salzsäuregehalt in Kubikzentimetern der verbrauchten $1 / 10$ n-Lauge.

B. v. $\mathrm{Beck}^{3}$ ) beobachtete eine Milzexstirpation, bei der bis 6 Wochen nach der Operation eine Vermehrung der weissen Blutkörperchen gefunden wurde. Desgleichen erwähnt $\left.\mathrm{O} . \mathrm{Vulpius}{ }^{4}\right)$ : „Es tritt bei den entmilzten Tieren eine vorübergehende absolute

1) Compt. rend. de la société de biol. Sér. X t. 4. 1897.

2) Beiträge zur klin. Chirurgie Bd. 63 H. 3.

3) Münchener mediz. Wochenschr. $1897 \mathrm{Nr} .47$.

4) Beiträge zur klin. Chirurgie Bd. 11. 1894. 
Milz und Magenverdauung und der angebliche Pepsingehalt der Milz. 599

Leukocytose auf, die bis zur Verdoppelung der normalen Durchschnittsmenge ansteigen kann, nach höchstens 9 Wochen aber ver-

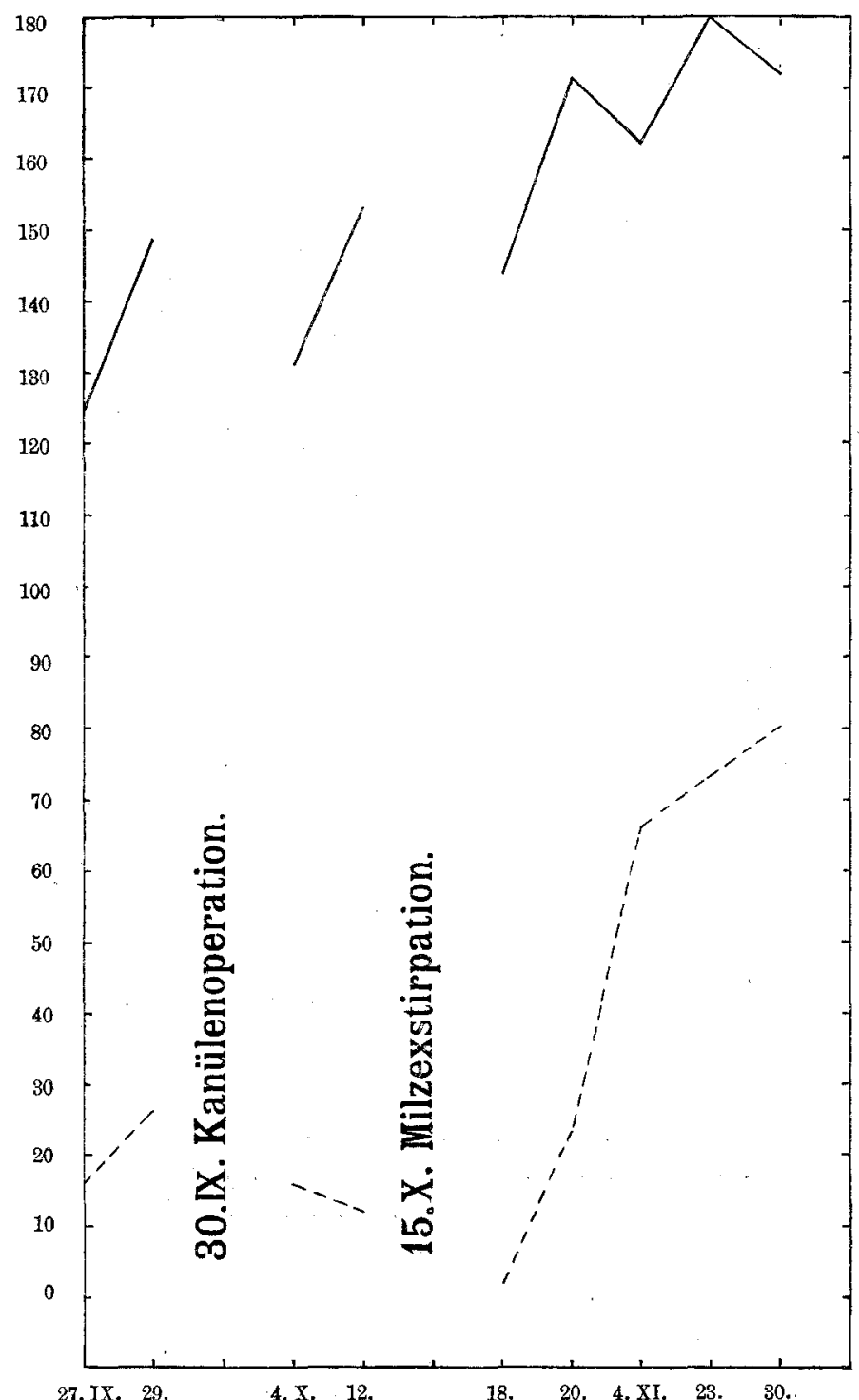

Fig. 2. Die Zahlengrösse der Kurve geben die im 1/100 qmm ausgezählten weissen Blutkörperchen an. $-=$ Leukocyten. - - - $=$ Lymphocyten.

schwindet." Gleiche Blutbefunde nach Milzexstirpation am Menschen sind von Péan, Credé, Czerny, Rigner u. a. beobachtet worden ${ }^{\mathbf{1}}$ ).

1) 0. Vulpius, l. c. 


\section{Versuchsserie II.}

Duodenalfistel. 8. November. Vorversuche 12 . Dezember 1910 bis 5. Januar 1911. Milzexstirpation 5. Januar. Versuche am entmilzten Versuchstier ab 10. Januar. Zum Versuch wird ein Bastard rom deutschen Schäferhund benutzt. Das Tier ist sehr ruhig und eignet sich daher gut zu dem Experiment. Ihm wird eine Kanüle in das Duodenum nahe dem Pylorus eingeführt.

Operation: Schnitt in der Linea alba. Das Duodenum wird vorgezogen, ca. $12 \mathrm{ccm}$ vom Pylorus durch einen Schnitt geöffnet, die Kanüle eingefübrt and in derselben Weise wie im vorhergehenden Versuch kurz hinter dem Pylorus durch die Darmwand und dam durch die Bauchwand geführt. Der Darm wird durch doppelte Naht verschlossen, ebenso die Bauchwunde. Zum oberflächlichen Schutz dient Kollodium.

Die Operation wird am 8. November in Äthermorphiumnarkose ausgeführt. Der Darm wird bei der Operation stark gezerrt, wir warten daher mit dem Beginn der Versuche bis zum 12. Dezember.

I.

Am 12. Dezember wird der Hund morgens nicht gefüttert.

$4 \mathrm{~h} 20^{\prime}$ trinkt er bei geöffneter Kanüle $50 \mathrm{ccm}$ Wasser, das in 15 Minuten klar abläuft.

$4^{\text {h }} 35^{\prime}$ bekommt der Hund $100 \mathrm{~g}$ sterilisiertes Fleisch $+30 \mathrm{ccm}$ Wasser zu fressen.

$4^{\mathrm{h}} 50^{\prime}$ erscheinen bereits geringe Fleischspuren in der durch die Kanüle ablaufenden wässerigen, zeitweise etwas gallig gefärbten Flüssigkeit.

$66^{\mathrm{h}} 25^{\prime}$ entleert sich durch die Kanüle dicker Fleischbrei.

$7 \mathrm{~h} 00^{\prime}$ scheint der Magen leer zu sein, da nur wenig getrübte Flüssigkeit abläuft.

Der Hund bekommt wieder Wasser zu trinken, das sich klar durch die Kanüle entleert.

Die Gesamtmenge des Mageninhalts, der von $4^{\mathrm{h}} 35^{\mathrm{l}}$ bis $7^{\mathrm{h}}$ durch die Kanüle abgegangen ist, beträgt $283 \mathrm{~g}$. Die Bestimmung des gelösten Stickstoffs nach Kjeldahl ergibt, dass gelöst sind:

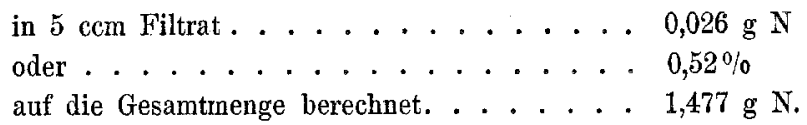

Freie Salzsäure war im Filtrat nicht vorhanden.

Die Azidität betrug 78 .

\section{II.}

$5^{\mathrm{h}} 10^{\prime}$ Beginn des Versuches. Der Hund trinkt $100 \mathrm{ccm}$ Wasser. In 15 Minuten laufen durch die Kanüle $150 \mathrm{ccm}$ klare Flüssigkeit ab. Diese hat eine Gesamtazidität von 19. Freie Salzsäure 10.

$5 \mathrm{~h} 25^{\prime}$ frisst der Hund $100 \mathrm{~g}$ sterilisiertes Fleisch $+30 \mathrm{ccm}$ Wasser.

5 h $35^{\prime}$. zeigen sich die ersten Fleischspuren.

6 h $10^{\prime}$ kommt dicker Fleischbrei aus der Kanüle.

$8^{\mathrm{h}} 00^{\prime}$ Schluss des Versuches. 
Es haben sich im ganzen durch die Kanüle entleert $325 \mathrm{~g} .10 \mathrm{ccm}$ werden zur Aziditätsbestimmung. verwandt.

Freie Salzsäure: keine.

Gesamtazidität 78.

Die übrigen $315 \mathrm{~g}$ werden mit gleichen Teilen einer 1/10 normal-Salzsäure versetzt. Hiervon werden $30 \mathrm{ccm}$ Filtrat hergestellt und $\mathrm{zu}$ je $10 \mathrm{ccm}$ für die Stickstoffbestimmung ǹach $\mathrm{Kjeldahl}$ abgemessen. $2 \times 5 \mathrm{ccm}$ Filtrat werden zur Verdauungsbestimmung durch Mett'sche Röhrchen verwandt. Der Rest wird in einem grösseren Gefäss zu 24 stündiger Nachverdauung in den Brutschrank gestellt. Temperatur $37^{\circ}$. Nach 24 Stunden wird wieder die Stickstoff bestimmung nach $\mathrm{Kjeldahl}$ ausgeführt.

Die Bestimmungen ergaben:

1. Vor der Nachverdauung sind gelöst:

in $10 \mathrm{ccm}$ Filtrat . . . . . . . $0,026 \mathrm{~g} \mathrm{~N}$

oder . . . . . . . . . . . $0,26 \%$

auf die Gesamtmenge berechnet. . . . . . 1,638 g N.

2. Nach der Nachverdauung sind gelöst:

in $10 \mathrm{ccm}$ Filtrat . . . . . . . . . $0,036 \mathrm{~g} \mathrm{~N}$

oder . . . . . . . . . . . . . $0,365 \%$

auf die Gesamtmenge berechnet. . . . . . 2,153 g N.

3. In den Mett'schen Röhrchen sind rach 24 Stunden $2,2 \mathrm{~mm}$ verdaut.

III.

2h $53^{\prime}$ Beginn des Versuches. Der Hund trinkt $100 \mathrm{ccm}$ Wasser. In 15 Minuten laufen durch die Kanüle $105 \mathrm{ccm}$ klare Flüssigkeit ab. Freie Salzsäure fehlt darin. Gesamtazidität 8.

3h $17^{\prime}$ frisst der Hund $100 \mathrm{~g}$ sterilisiertes Fleisch mit $30 \mathrm{ccm}$ Wasser angerührt. $3^{\mathrm{h}} 30^{\prime}$ zeigen sich in der ablaufenden Flüssigkeit die ersten Fleischspuren.

$4 \mathrm{~h} 30^{\prime}$ entleert sich aus der Kanüle dicker Fleischbrei.

$6 \mathrm{~h} 00^{\prime}$ Schluss des Versuches.

Es sind im ganzen durch die Kanüle abgelaufen $315 \mathrm{~g}$. $10 \mathrm{ccm}$ werden zur Aziditätsbestimmung benutzt.

Gesamtazidität 64 .

Freie Salzsäure fehlt.

Wie im vorigen Versuch werden die übrigbleibenden $305 \mathrm{~g}$ zu gleichen Teilen mit $1 / 10$ normal-Salzsäure versetzt, daron $30 \mathrm{ccm}$ Filtrat zur Stickstoffbestimmung und $10 \mathrm{ccm}$ für die Mett'schen Röhrchen abgenommen. Der Rest wird zu 24 stündiger Nachverdaung in den Brutschrank gestelit. Temperatur $37^{\circ}$. Nach 24 Stunden wird wieder der gelöste Stickstoff bestimmt.

Die Bestimmungen ergaben:

1. Vor der Nachverdauung sind gelöst:

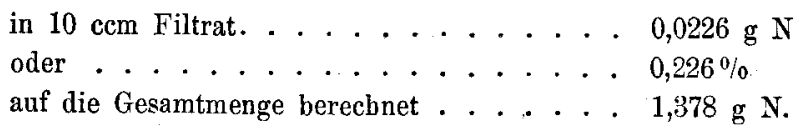


2. Nach der Nachverdauung sind gelöst:

in $100 \mathrm{ccm}$ Filtrat ....... . . 0,0329 $\mathrm{g} \mathrm{N}$

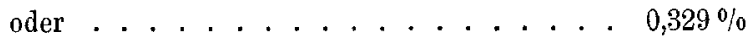

auf die Gesamtmenge berechnet . . . . . 1,875 g N.

3. In den Mett'schen Röhrchen sind nach 24 Stunden nur 0,7 mm verdaut.

In derselben Weise wie mit dem sterilisierten Fleisch wurden die Versuche mit getrocknetem Weissbrot ausgeführt.

\section{IV.}

$4 \mathrm{~h} 15^{\prime}$ Beginn des Versuches. Der Hund trinkt $100 \mathrm{ccm}$ Wasser. In 15 Minuten laufen $110 \mathrm{ccm}$ klare Flüssigkeit durch die Kanüle ab. Dieselbe enthält keine freie Salzsäure. Gesamtazidität ist 3.

$4^{\mathrm{h}} 30^{\prime}$ frisst der Hund $25 \mathrm{~g}$ trockenes gemahlenes Weissbrot $+50 \mathrm{ccm}$ Wasser.

$5^{\mathrm{h}} 15^{\prime}$ zeigen sich die ersten Brotspuren.

$5 \mathrm{~h} 30^{\prime}$ entleert sich dicker Brotbrei aus der Kanüle.

$66^{\text {h }} 40^{\prime}$ Schluss des Versuches.

Die Gesamtmenge der entleerten Massen beträgt $151 \mathrm{~g} .10 \mathrm{ccm}$ dienen zur Aziditätsbestimmung.

Gesamtazidität 47.

Freie Salzsäure fehlt.

Die übrigbleibenden $141 \mathrm{~g}$ des Mageninhalts werden mit einer gleichen Menge $1 / 10$ normal-Salzsäure versetzt und hiervon $20 \mathrm{ccm}$ Filtrat für Stickstoffbestimmung nach $\mathrm{Kjeldahl}$ und $10 \mathrm{ccm}$ Filtrat für Mett'sche Röhrchen hergestellt. Der Rest kommt zur Nachverdauung auf 24 Stunden in den Brutschrank. Temperatur $37^{\circ}$ C. Nach 24 Stunden wird wieder die Stickstoffbestimmung ausgeführt.

Die Bestimmungen ergeben:

1. Vor der Nachverdauung sind gelöst:

in $100 \mathrm{ccm}$ Filtrat. . . . . . $0,01 \mathrm{~g} \mathrm{~N}$

oder .................... $0,105 \%$

auf die Gesamtmenge berechnet. . . . . $0,296 \mathrm{~g} \mathrm{~N}$.

2. Nach der Nachverdauung sind gelöst:

in $100 \mathrm{ccm}$ Filtrat. . . . . . . . 0,016 g N

oder................. . . $0,165 \%$

auf die Gesamtmenge berechnet. . . . . 0,415 g N.

3. In den Mett'schen Röhrchen sind nach 24 Stunden $3,0 \mathrm{~mm}$ verdaut.

V.

$11^{\text {h }} 35^{*}$ Beginn des Versuches. Der Hund trinkt $80 \mathrm{ccm}$ Wasser. In 15 Minuten entleeren sich durch die Kanüle $285 \mathrm{ccm}$ klare Flüssigkeit. Gesamtazidität 35. Freie Salzsäure 9 .

11 h $50^{\prime}$ frisst der Hund $25 \mathrm{~g}$ trockenes gemahlenes Weissbrot $+50 \mathrm{ccm}$ Wasser. $12 \mathrm{~h} 05^{\prime}$ erscheinen die ersten Brotspuren.

$12^{\mathrm{h}} 15^{\prime}$ entleert sich dicker Brotbrei.

$1^{\text {h }} 00^{\prime}$ Schluss des Versuches. 
Milz und Magenverdauung und der angebliche Pepsingehalt der Milz. 603

Die Gesamtmenge des entleerten Mageninhalts beträgt $273 \mathrm{~g} .10 \mathrm{ccm}$ wurden zur Säurebestimmung verwandt.

Gesamtazidität 67.

Freie Salzsäure fehlt.

Wie im vorhergehenden Versuch wird mit dem Rest eine Nachverdauung im Brutschrank nach Zusatz von gleichen Teilen 1/10 normal-Salzsäure vorgenommen und vor und nach dem Aufenthalt im Brutschrank die Stickstoffbestimmung nach Kjeldahl ausgeführt. Ausserdem, wird ein Versuch mit Mett'schen Röhrchen angesetzt.

Die Bestimmungen ergaben:

1. Vor der Nachverdauung sind gelöst:

$$
\begin{aligned}
& \text { in } 10 \mathrm{ccm} \text { Filtrat . . . . . . . . } 0,007 \mathrm{~g} \mathrm{~N} \\
& \text { oder ................. . . } 0,071 \% \\
& \text { auf die Gesamtmenge berechnet . . . . . 0,373 g N. }
\end{aligned}
$$

2. Nach der Nachverdauung sind gelöst:

in $10 \mathrm{ccm}$ Filtrat . . . . . . . . $0,01 \mathrm{~g} \mathrm{~N}$

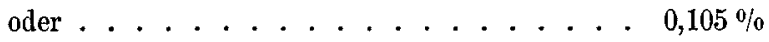

auf die Gesamtmenge berechnet. . . . . 0, 0,52 g N.

3. In den Mett'schen Röhrchen sind nach 24 Stunden $3,6 \mathrm{~mm}$ verdaut.

Die Blutuntersuchung zeigt ein Schwanken der absoluten Anzahl weisser Blutkörperchen zwischen 13000 und 17000 im Kubikmillimeter. Der prozentuale Gehalt an Lymphocyten bewegt sich zwischen $13 \%$ und $18 \%$. Auffallend ist eine grössere Zahl eosinophiler Leukocyten. Ihre Menge schwankt zwischen $3 \%$ und $9 \%$.

Am 5. Januar wird der Hund in Morphiumäthernarkose entmilzt. Er äbersteht die Operation gut. Am 10. Januar ist er bereits wieder so weit hergestellt, dass mit den Versuchen begonnen werden kann.

\section{VI.}

$3^{\mathrm{h}} 20^{\prime}$ Beginn des Versuches. Der Hund trinkt $90 \mathrm{ccm}$ Wasser. In 15 Minuten fliessen durch die Kanüle $105 \mathrm{ccm}$ klare Flüssigkeit ab. Freie Salzsäure ist darin nicht vorhanden. Gesamtazidität 3,5.

$3 \mathrm{~h} 35^{\prime}$ frisst der Hund $100 \mathrm{~g}$ sterilisiertes Fleisch $+30 \mathrm{ccm}$ Wasser.

4h $05^{\prime}$ erscheinen die ersten Fleischspuren.

$4^{\text {h }} 30^{\prime}$ entleert sich dicker Fleischbrei.

5 h $30^{\prime}$. Schluss des Versuches.

Gesamtmenge des entleerten Mageninhalts $311 \mathrm{~g} .10 \mathrm{ccm}$ zur Aziditätsbestimmung.

Gesamtazidität 98.

Freie Salzsäure fehlt.

Die weitere Behandlung des gewonnenen Mageninhalts sowie die Verdauungsbestimmungen geschehen wie vor der Entmilzung. 
Die Bestimmungen ergaben:

1. Vor der Nachverdauung sind gelöst:

in $10 \mathrm{ccm}$ Filtrat ......... $0,023 \mathrm{~g} \mathrm{~N}$

oder . . . . . . . . . . . . . . . . . . $0,231 \%$

auf die Gesamtmenge berechnet. . . . . . . 1,39 g N.

2. Nach der Nachverdauung sind gelöst:

in $10 \mathrm{ccm}$ Filtrat . . . . . . . 0,035 $\mathrm{g} \mathrm{N}$

oder....................... $0,358 \%$

auf die Gesamtmenge berechnet. . . . . . 2,047 g N.

3. In den Mett'schen Röhrchen sind verdaut in 24 Stunden $2,3 \mathrm{~mm}$.

VII.

$2 \mathrm{~h} 20^{\prime}$ Beginn des Versucbes. Der Hund trinkt nur $10 \mathrm{ccm}$ Wasser und ist zum Weitertrinken nicht $\mathrm{zu}$ bewegen. Wir warten 15 Minuten. Es entleert sich etwas klare Flüssigkeit aus der Kanüle.

2 h $35^{\prime}$ frisst der Hund $25 \mathrm{~g}$ trockenes, gemahlenes Weissbrot $+50 \mathrm{ccm}$ Wasser.

2 h $50^{\prime}$ die ersten Brotspuren.

$3 \mathrm{~h} 15^{\prime}$ entleert sich aus der Kanüle dicker Brotbrei.

4 h $00^{\prime}$ Schluss des Versuches.

Gesamtmenge des entleerten Mageninhalts $165 \mathrm{~g}$. $10 \mathrm{ccm}$ dienen zur Säurebestimmung.

Gesamtazidität 39.

Freie Salzsäure fehlt.

Es folgt wie in den früheren Versuchen eine 24 stündige Nachverdauung im Brutschrank nach vorherigem Zusatz von gleichen Teilen 1/10 normal-Salzsäure. Vor und nach der Nachverdauung wird die Stickstoffbestimmung ausgeführt, ebenso ein Versuch mit Mett'schen Röhrchen angesetzt.

Die Bestimmungen ergaben:

1. Vor der Nachverdauung sind gelöst:

in $10 \mathrm{ccm}$ Filtrat . . . . . . . 0,008 $\mathrm{g} \mathrm{N}$

oder................ $0,084 \%$

auf die Gesamtmenge berechnet. . . . . . $0,26 \mathrm{~g} \mathrm{~N}$.

2. Nach der Nachverdauung sind gelöst:

in $10 \mathrm{ccm}$ Filtrat ......... . . $0,013 \mathrm{~g} \mathrm{~N}$

oder................. $0,138 \%$

auf die Gesamtmenge berechnet. . . . . . . 0,386 g N.

3. In den Mett'schen Röhrchen sind in 24 Sţunden $3,2 \mathrm{~mm}$ verdaut.

VIII.

$2^{\text {h }} 10^{\prime}$ Beginn des Versuches. Der Hund ist zum Wassertrinken nicht zu bewegen. Wir warten 15 Minuten. Es entleert sich etwas Flüssigkeit aus der Kanüle.

$2 \mathrm{~h} 25^{\prime}$ frisst der Hund $25 \mathrm{~g}$ getrocknetes, gemahlenes Weissbrot $+50 \mathrm{ccm}$ Wasser.

$2^{\text {h }} 35^{\prime}$ zeigen sich die ersten Brotspuren. 
Milz und Magenverdauung und der angebliche Pepsingehalt der Milz. 605 2 h $55^{\prime}$. entleert sich dicker Brotbrei aus der Kanüle.

3 h $40^{\prime}$ Schluss des Versuches.

Die Gesamtmenge des gewonnenen Mageninhalts ist $152 \mathrm{~g} .10 \mathrm{ccm}$ dienen zur Säurebestimmung.

Gesamtazidität 20.

Freie Salzsäure fehlt.

Die weitere Behandlung des Mageninhalts geschieht wie im vorhergehenden Versuch, ebenso die Verdaunngshestimmungen.

Die Bestimmungen ergaben:

1. Vor der Nachverdauung sind gelöst:

in $10 \mathrm{ccm}$ Filtrat . . . . . . . . $0,009 \mathrm{~g} \mathrm{~N}$

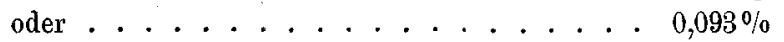

auf die Gesamtmenge berechnet. . . . . 0,266 g N.

2. Nach der Nachrerdaunng sind gelöst:

in $10 \mathrm{ccm}$ Filtrat . . . . . . . . $0,016 \mathrm{~g} \mathrm{~N}$

oder ............. . . $0,161 \%$

auf die Gesamtmenge berechnet . . . . . . $0,408 \mathrm{~g} \mathrm{~N}$.

3. In den Mett'schen Röhrchen sind in 24 Stunden $3,5 \mathrm{~mm}$ verdaut.

\section{XI.}

1h $35^{\prime}$ Beginn des Versuches. Der Hund trinkt $90 \mathrm{ccm}$ Wasser. In 15 Minuten fliessen durch die Kanüle $100 \mathrm{ccm}$ ab. Freie Salzsäure fehlt darin. Gesamtazidität 6 .

1 h $50^{\prime}$ frisst der Hund $100 \mathrm{~g}$ sterilisiertes Fleisch $+30 \mathrm{ccm}$ Wasser.

2 h $00^{\prime}$ zeigen sich die ersten Fleischspuren.

2 h $35^{\prime}$ ' entleert sich dicker Fleischbrei aus der Kanüle.

3 h $20^{\prime}$ Schluss des Versuches.

Gesamtmenge des entleerten Mageninhalts $289 \mathrm{~g}$. Mit $10 \mathrm{ccm}$ wird die Azidität bestimmt.

Gesamtazidität 90.

Freie Salzsäure fehlt.

Die übrigbleibenden $279 \mathrm{~g}$ werden in derselben Weise wie in den vorhergehenden Versuchen zu gleichen Teilen mit $1 / 10$ normal-Salzsäure versetzt und zu 24 stündiger Nachverdauung aufgestellt. Vorher und nachher wird die Stickstoffbestimmung nach $\mathrm{Kj}$ eldahl ausgeführt. Ebenso werden Mett'sche Röhrchen angesetzt.

Die Bestimmungen ergaben:

1. Vor der Nachverdauung sind gelöst:

in $10 \mathrm{ccm}$ Filtrat . . . . . . . . $0,022 \mathrm{~g} \mathrm{~N}$

oder ................ . . . $0,226 \%$

auf die Gesamtmenge berechnet. . . . . . 1,261 g N.

2. Nach der Nachverdauung sind gelöst:

in $10 \mathrm{ccm}$ Filtrat . . . . . . . . $0,037 \mathrm{~g} \mathrm{~N}$

oder ............... . . 0,371\%

auf die Gesamtmenge berechnet. . . . . . 1,953 g N.

3. In den Mett'schen Röhrchen sind in 24 Stunden $3,4 \mathrm{~mm}$ verdaut 
Die Untersuchung des.Blutes nach der Entmilzung zeigte einen Anstieg der weissen Blutkörperchen von 13600 bis $18000 \mathrm{im}$ Kabikmillimeter. Der prozentuale Gehalt an Lymphocyten betrug $21 \%$ und dann $26 \%$. Auf 100 Lenkocyten kamen sechs Eosinophile.

Es fand sich also auch nach Ausschaltung der Zufälligkeiten, wie sie in Versuch I möglich waren, keine Beeinträchtigung der Magenverdauung nach Milzexstirpation. Wir fanden die Menge des gelösten Stickstoffs im Filtrat des quantitativ entleerten Mageninhalts sowohl prozentual als auch auf die Gesamtmenge berechnet innerhalb derselben Grenzen sehwanken wie vor der Entmilzung. Eine Übersicht der gefundenen Werte gibt die nachfolgende Tabelle:

Vor der Entmilzung.

\begin{tabular}{|c|c|c|c|c|c|}
\hline \multicolumn{6}{|c|}{ Versuche mit Fleisch. } \\
\hline \multirow{2}{*}{$\begin{array}{l}\text { Säure- } \\
\text { gehalt }\end{array}$} & \multirow{2}{*}{$\begin{array}{c}\text { Mett'sche } \\
\text { Röhrchen } \\
24 \text { Stdn. }\end{array}$} & \multicolumn{2}{|c|}{$\begin{array}{c}\text { Gelöster Stickstoff } \\
\text { vor der Nachverdauung }\end{array}$} & \multicolumn{2}{|c|}{$\begin{array}{c}\text { Gelöster Stickstoff } \\
\text { nach der Nachverdauung }\end{array}$} \\
\hline & & in $10 \mathrm{ccm}$ & $\begin{array}{c}\text { auf die } \\
\text { Gesamtmenge } \\
\text { berechnet }\end{array}$ & in $10 \mathrm{ccm}$ & $\begin{array}{c}\text { auf die } \\
\text { Gesamtmenge } \\
\text { berechnet }\end{array}$ \\
\hline $\begin{array}{l}78 \\
64\end{array}$ & $\begin{array}{l}2,2 \mathrm{~mm} \\
0,7 \mathrm{~m}\end{array}$ & $\begin{array}{l}0,026 \mathrm{~g} \mathrm{~N} \\
0,0226 \mathrm{~g} \mathrm{~N}\end{array}$ & $\begin{array}{l}1,638 \mathrm{~g} \mathrm{~N} \\
1,378 \% "\end{array}$ & $\begin{array}{l}0,036 \mathrm{~g} \mathrm{~N} \\
0,0329 \mathrm{~g} \mathrm{~N}\end{array}$ & $\begin{array}{l}2,153 \mathrm{~g} \mathrm{~N} \\
1,875 " \#\end{array}$ \\
\hline $\begin{array}{l}98 \\
90\end{array}$ & $\begin{array}{l}2,3 \mathrm{~mm} \\
3,4,\end{array}$ & $\begin{array}{c}\text { Nach de } \\
0,023 \mathrm{~g} \mathrm{~N} \\
0,022 " \text { " } \\
\text { Vor der }\end{array}$ & 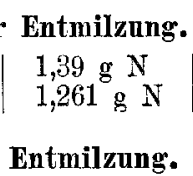 & $\begin{array}{l}0,035 \mathrm{~g} \mathrm{~N} \\
0,037 " \#\end{array}$ & $\begin{array}{l}2,047 \cdot \mathrm{g} \mathrm{N} \\
1,953 " \eta\end{array}$ \\
\hline
\end{tabular}

\begin{tabular}{|c|c|c|c|c|c|}
\hline \multicolumn{6}{|c|}{ Versuche mit Brot. } \\
\hline \multirow[b]{2}{*}{$\begin{array}{l}\text { Säure- } \\
\text { gehalt }\end{array}$} & \multirow{2}{*}{$\begin{array}{l}\text { Mett'sche } \\
\text { Röhrchen } \\
24 \text { Stdn. }\end{array}$} & \multicolumn{2}{|c|}{$\begin{array}{l}\text { Gelöster Stickstoff } \\
\text { vor der Nachverdauung }\end{array}$} & \multicolumn{2}{|c|}{$\begin{array}{c}\text { Gelöster Stickstoff } \\
\text { nach der Nachverdauung }\end{array}$} \\
\hline & & in $10 \mathrm{ccm}$ & $\begin{array}{c}\text { auf die } \\
\text { Gesamtmenge } \\
\text { berechnet }\end{array}$ & in $10 \mathrm{cem}$ & $\begin{array}{c}\text { auf die } \\
\text { Gesamtmenge } \\
\text { berechnet }\end{array}$ \\
\hline $\begin{array}{l}47 \\
67\end{array}$ & $\begin{array}{l}3,0 \mathrm{~mm} \\
3,6 \quad "\end{array}$ & $\begin{array}{l}0,01 \mathrm{~g} \mathrm{~N} \\
0,007 \mathrm{~g} \mathrm{~N}\end{array}$ & $\begin{array}{l}0,296 \mathrm{~g} \mathrm{~N} \\
0,373 \Rightarrow "\end{array}$ & $\begin{array}{l}0,016 \mathrm{~g} \mathrm{~N} \\
0,01 \mathrm{~g} \mathrm{~N}\end{array}$ & $\begin{array}{l}0,415 \mathrm{~g} \mathrm{~N} \\
0,52 \mathrm{~g} \mathrm{~N}\end{array}$ \\
\hline \multicolumn{6}{|c|}{ Nach der Entmilaung. } \\
\hline $\begin{array}{l}39 \\
20\end{array}$ & $\begin{array}{l}3,2 \mathrm{~mm} \\
3,5 \quad "\end{array}$ & $\begin{array}{l}0,008 \mathrm{~g} \mathrm{~N} \\
0,009 \Rightarrow, n\end{array}$ & $\begin{array}{l}0,26 \mathrm{~g} \cdot \mathrm{N} \\
0,266 \mathrm{~g} \mathrm{~N}\end{array}$ & $\begin{array}{l}0,013 \mathrm{~g} \mathrm{~N} \\
0,016 \% "\end{array}$ & $\begin{array}{l}0,386 \mathrm{~g} \mathrm{~N} \\
0,408 \%\end{array}$ \\
\hline
\end{tabular}

\section{Versuchsserie III.}

Am 5. November wird einem Bastard vom deutschen Schäferhund durch einen Schnitt in der Linea alba die Bauchböhle eröffnet und aus der Pylorusund Fundusgegend des Magens je ein Stück Schleimhaut ausgeschnitten. Die 
Milz und Magenverdauung und der angebliche Pepsingehalt der Milz. 607

Wundränder werden durch. doppelte Nähte wieder vereinigt, dann wird die Milz exstirpiert und nach sorgfältiger Versorgung des Stumpfes die Bauchwunde wieder geschlossen. Die Operation fand am Nachmittag statt in Morphiumäthernarkose. Der Hund hat seit dem Abend zu vor nichts gefressen.

Die beiden exstirpierten Schleimhautstücke wogen: aus dem Fundusteil. . . . . . . . 4, $4,3 \mathrm{~g}$ aus dem Pylorusteil ......... . 5,2 g

Jedes Stück wird im Mörser mit Kieselguhr zerrieben, mit dem fünffachen Volumen $1 / 10$ normal-Salzsäure versetzt und auf eine Stunde bei $38^{\circ} \mathrm{C}$. in den Brutschrank gestellt. Darauf werden die beiden Infuse abfiltriert und zwei Mett'sche Röhrchen mit dem Filtrat angesetzt.

In 24 Stunden verdaut:

das Infus aus der Schleimhaut des Fundusteils . . . . . . 2,6 mm das Infus aus der Schleimhaut des Pylorusteils . . . . . 5,4 mm.

Am 21. November wird der Hund in Morphiumäthernarkose durch Pneumothorax ad exitum gebracht. Er hat wieder seit dem Abend zuvor nichts gefressen.

Die Innenfläche der Magenschleimhaut zeigt zwei gut verheilte Narben in der Fundus- und Pylorusgegend. Es werden neben diesen Narben mit Vermeidung der Narbenwülste zwei Schleimbautstücke ausgeschnitten.

Die Schleimhautstücke wogen:

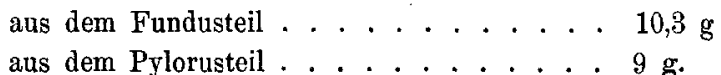

Die beiden Schleimhantstücke werden in der vorher beschriebenen Weise behandelt.

In 24 Stunden verdaut:

das Infus aus der Schleimhaut des Fundusteils . . . . . . 4,0 mm

das Infus aus der Schleimhaut des Pylorusteils . . . . . . . 5,1 mm.

Es trat also nach der Exstirpation der Milz keine Abnahme des Pepsingehalts der Magenschleimhaut ein. Derselbe ist im Fundusteil sogar erhöht.

\section{Versuchsserie IV.}

Wie bereits zu Beginn dieser Arbeit erwähnt wurde, haben Ebstein und Grützner darauf hingewiesen, dass bei Extraktionen der Schleimhaut mit geringen Flüssigkeitsmengen während kurzer Zeit wesentlich nur das freie Pepsin im Infus gewonnen wird, dieses freie Pepsin aber keineswegs parallel dem Gesamtgehalt an freiem und gebundenem Pepsin geht.

Um einem derartigen Einwurf zu entgehen - wir sind der Überzeugung, ihn bereits dadurch unmöglich gemacht zu haben, dass wir die Magenschleimhaut mit Kieselgur fein zerrieben -, wird bei den nachfolgenden Versuchen mit dem Magen milzexstirpierter Ratten eine grössere Menge Salzsäure (das 40 fache Volumen) zur Her- 
stellung des Infuses verwandt und der Aufenthalt im Brutschrank auf 24 Stunden ausgedehnt.

Von zwanzig Versuchsratten, die unter denselben Lebensbedingungen in einem Käfig hausen, wird am 2. Dez. in Äthernarkose zehn die Milz exstirpiert. Vier sterben nach 24 Stunden. Am 9. Dez. stirbt die fünfte.

Am 20. Dez. wird weiteren drei Ratten die Milz exstirpiert. Davon bleibt nur eine am Leben.

Von drei gestorbenen Ratten wird gleichfalls ein Infus des Magens hergestellt.

Die gewonnenen Resultate gehen aus der nachfolgenden Tabelle hervor. Die Verdauungsgrösse ist durch Mett'sche Röhrchen bestimmt und in Millimeter ausgedrückt.

\begin{tabular}{|c|c|c|c|c|c|c|c|c|c|c|}
\hline \multicolumn{5}{|c|}{ Entmilzte Ratten } & $\longleftrightarrow$ & \multicolumn{5}{|c|}{ Kontrolltiere } \\
\hline \multicolumn{3}{|c|}{$\begin{array}{l}\text { Dauer der Ver- } \\
\text { dauung }\end{array}$} & \multirow{2}{*}{ 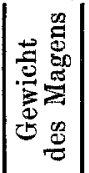 } & \multirow{2}{*}{$\begin{array}{l}\text { Füllung } \\
\text { des } \\
\text { Magens }\end{array}$} & \multirow{2}{*}{$\begin{array}{c}\text { Datum } \\
\text { des } \\
\text { Versuches }\end{array}$} & \multirow{2}{*}{$\begin{array}{l}\text { Fullung } \\
\text { des } \\
\text { Magens }\end{array}$} & \multirow{2}{*}{ 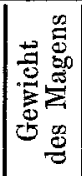 } & \multicolumn{3}{|c|}{$\begin{array}{l}\text { Dauer der Ve'- } \\
\text { dauung }\end{array}$} \\
\hline $\begin{array}{c}144 \\
\text { Stdn. }\end{array}$ & $\begin{array}{c}46 \\
\text { Stdn. }\end{array}$ & $\mid \begin{array}{c}24 \\
\text { Stdn. }\end{array}$ & & & & & & $\begin{array}{c}24 \\
\text { Stdn. }\end{array}$ & $\begin{array}{c}46 \\
\text { Stdn. }\end{array}$ & $\begin{array}{c}144 \\
\text { Stdn. }\end{array}$ \\
\hline
\end{tabular}

Entmiltzt am 2. Dezember.

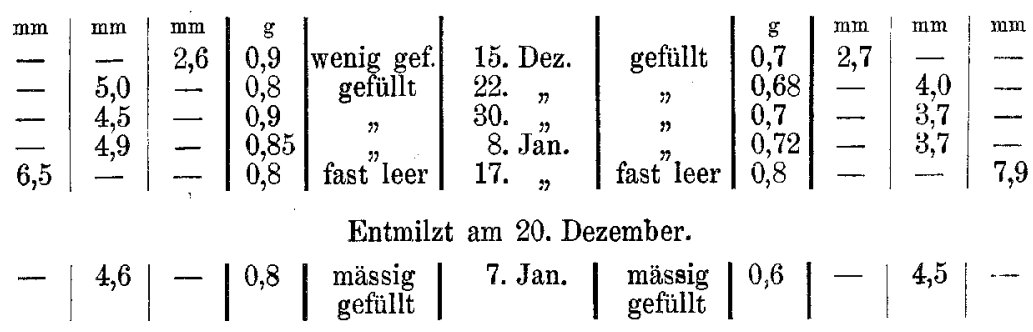

Gestorbene Ratten.

\begin{tabular}{l|c|c|c}
\hline \hline Datum der Entmilzung & $\begin{array}{c}\text { Gewicht } \\
\text { des Magens }\end{array}$ & \multicolumn{2}{|c}{ Dauer der Verdauung } \\
\cline { 3 - 4 } & 24 Stunden & 46 Stunden \\
\hline & & $\mathrm{mm}$ & $\mathrm{mm}$ \\
2. Dezember entmilzt, 9. Dezember + & 0,7 & 2,9 & $\overline{\mathbf{4 , 5}}$ \\
20. Dezember entmilzt, 20. Dezember $\dagger$ & 0,8 & - & 4,4
\end{tabular}

Die Resultate unserer Versuche stehen in direktem Gegensatz zu den Versuchen von Tarulli und Paskucci. Wir haben in allen vier Versuchen übereinstimmend keine Herabsetzung der Magenverdaung nach Milz- 
Milz und Magenverdaung und der angebliche Pepsingehalt der Milz. 609 exstirpation konstatieren können. Die stets gleichen Resultate bei völliger Verschiedenheit in der Anordnung der Versuche lassen uns einen Versuchsfehler ausschliessen. Tarulli und Paskucci haben stets dieselbe Methode benutzt, und es ist anzunebmen, dass in der Art ihrer Bestimmung der Grund für die erhaltenen abweichenden Werte liegt.

Der Gross'sche Versuch am Menschenkann bei den komplizierten Verhältnissen (Trauma, psychische Beeinflussung durch Sondenausheberung, an die der Patient erst nach der Milzexstirpation gew öht wurde usw.) keineswegs Anspruch darauf machen, als Beweis für einen Einfluss der Milz auf die Magenverdaung $z u$ gelten.

Die Resultate werden durch die theoretischen Betrachtungen, die Gross an seine Versuche knüpft, nicht wertvoller, zumal er sich bei diesen Betrachtungen auf den längst widerlegten ${ }^{1}$ ) Einfluss der Milz auf die Pankreasverdaung stützt.

\section{Versuche mit Milzinfus.}

Da wir, wie aus den vorhergehenden Versuchen hervorgeht, keinen Einfluss der Milz auf die Verdaungstätigkeit des Magens bei Hunden und Ratten hatten konstatieren können, so interessierte es uns auch, die Versuche von Lo Monako und Tarulli²) nachzuprüfen, die im Laboratorium Lu ciani's ausgeführt worden sind. Die Resultate dieser Versuche wurden im Jahre 1901 auf dem Internationalen Physiologenkongress in Turin mitgeteilt. Luciani fasst dieses Resultat in den Worten zusammen: "dass das Infus der angeschwollenen Milz Pepsin enthält oder wenigstens ein Enzym, welches auf Fibrin in einem sauern Medium die gleiche verdauende Wirkung ausübt."

Leuret und Lassaigne ${ }^{3}$ ) wiesen im Jahre 1825 nach, dass die Milz bei Hunden, Katzen, Meersehweinchen und Kaninchen dann

1) 0. Prym, 1. c.

2) Luciani, Lehrbuch der Physiologie des Menschen S. 101.

3) Zitiert nach Schiff, Über die Funktion der Milz. Schweizerische Zeitschr. f. Heilkunde 1862. 
anschwillt und blutreicher wird, wenn der Darm anfängt die Verdauungsprodukte aus dem Magen aufzunehmen und die Lymphgefässe des Mesenteriums sicb füllen.

Dobson ${ }^{1}$ ) fand bei Hunden in der fünften Stunde nach der Mahlzeit das Maximum der Milzanschwellung. In der zwölften Stunde war die Milz wieder schlaff, klein und blutarm.

Dieselben Resultate hatten die Untersuchungen von Giesker, Landis, Bardeleben, Schönfeld u. a. ${ }^{1}$ ).

Diesen übereinstimmenden Resultaten stehen die Angaben von Gray ${ }^{1}$ ) entgegen, welcher die Milz erst 15 Stunden nach der Mahlzeit ihr böchstes Gewicht erreichen lässt.

E. Oehl ${ }^{2}$ ) hat diese Wahrnehmung der Milzanschwellung nicht bestätigen können. Er stellte fest, dass bei sieben grösseren und kieineren Hunden, die in der Verdauung begriffen waren, das Verhältnis des Körpergewichts zu dem der Milz zwischen 160:1 und $576: 1$ schwankte. Bei 16 nüchternen Hunden von verschiedener Grösse schwankte dieses Verhältnis zwischen 228:1 und 621:1, also in beiden Kategorien etwa um das Dreifache. $\mathrm{Oehl}$ erwähnt besonders, dass er trotz lebhafter Verdauung bei den gefütterten Hunden kein günstigeres Verhältnis von Milz zu Körpergewicht gefunden hat als bei den nüchternen.

Über die Versuchsanordnung der Experimente von Lo Monako und Tarulli sagt Luciani: „Man exstirpiert bei einem Hunde während voller Verdaungstätigkeit die Milz und teilt sie in kleine Stücke, die dann in einem Mörser mit Glaspulver zerrieben werden. Der so erhaltene Brei wird in einen Kolben gebracht und das fünffache Volumen $4 \%$ iger Borsäure zugesetzt. Man lässt das Infus durch 6 Stunden im Ofen bei $37^{\circ}$ stehen, dann filtriert man und erhält ein von einer durchsichtigen dunkelroten Flüssigkeit dargestelltes Infus. Von diesem Infus bringt man zwei gleiche Portionen von je $15 \mathrm{ccm}$ in zwei kleine Kolben und fügt $15 \mathrm{ccm} 4 \%$ ige Salzsäure und $1 / 2 \mathrm{~g}$ rohes Fibrin hinzu, das bereits durch die Einwirkung 2\%oiger Salzsäure, in der man es durch 30 Minuten in der Kälte stehen liess, gequollen war.

Um die Wirkung des angesäuerten Milzinfuses mit jenem zu

1) Zitiert nach $\mathrm{Sch}$ iff, Über die Funktion der Milz. Schweizerische Zeitschr. f. Heilkunde 1862.

2) E. Oehl, Gagg. Loml. 9. Okt. 1868, zitiert nach Schmidt's Jahrbücher. 
vergleichen, welche die Salzsäure allein auf das rohe Fibrin ausübt, bringt man die gleiche Menge aufgequollenes Fibrin mit $15 \mathrm{ccm}$ $4 \%$ iger Borsäure und $15 \mathrm{ccm} 4 \%$ iger Salzsäure in zwei weitere Kolben. Lässt man nun den Inhalt der vier Kolben bei $39^{\circ}$ stehen, so beobachtet man nach 2 Stunden, dass das Fibrin mit dem Milzinfus fast bis zur Hälfte verdaut ist, während jenes mit der Salzsäure allein keine Spur von Verdauung zeigt. Nach $3^{1 / 2}$ Stunden nimmt man die beiden anderen Kolben heraus und beobachtet, dass das Milzinfus das Fibrin fast vollständig verdaut hat, während an dem in der einfachen saueren Lösung gehaltenen Fibrin keine Andeutung einer Verdauung wahrnehmbar ist."

Ähnliche Untersuchungen auf ein proteolytisches Enzym der Milz sind von $\mathrm{Hedin}$ und $\mathrm{Row}$ land ${ }^{1}$ ) im Inner-Institut in London angestellt worden. Die beiden Forscher benutzten frische Schlachthausmilzen, die in einem besonderen von $\mathrm{Row}$ land konstruierten Apparat zu Brei gequetscht und nachher in einer bydraulischen Presse ausgepresst wurden. Die verdauende Wirkung des mit Salzsäure oder Essigsäure verdünnten Presssaftes wurde durch den Fortschritt der Autolyse oder durch seine Einwirkung auf Blutfibrin bestimmt. Die Stärke der Proteolyse wurde durch Bestimmung des gelösten Stickstoffs nach Kjeldahl festgestellt.

Hedin und Rowland fanden eine Einwirkung des Enzyms auf die im Milzsaft selbst befindlichen Eiweissstoffe und eine auflösende Wirkung auf Blutfibrin. Am stärksten wirkte jedesmal das Enzym in sauerer Lösung.

Dieselben Versuche, wie Hedin und Rowland, führte Leathes ${ }^{2}$ ) mit dem Presssaft der Ochsenmilz aus. Er trennte die gewonnenen Verdaungsprodukte nach $\mathrm{Kossel}$ und Kutscher und fand dieselben Produkte, wie nach Trypsinverdauung und Säurehydrolyse.

Die komplizierte Apparatur von Hedin und Row land stand uns nicht zur Verfügung. Wir hielten uns in unseren Versuchen an die Anordnung Luciani's. Wir taten dieses auch hauptsächlich darum, weil Luciani aus seinen Versuchen schliesst, dass die Milz Pepsin oder ein pepsinähnliches Enzym enthält, und Gross auf diesen Schluss hin einige Theorien entwirft, die diesen etwaigen

1) Zeitschr. f. physiol. Chemie Bd. 32 S. 3 und 4. 1901.

2) Journ. of Physiol. Bd. 28 S. 5. 12. Sept. 1902. 
Pepsingehalt mit der von ihm beobachteten Abnahme des Magenpepsins nach Milzexstirpation in Verbindung zu bringen versuchen. Eine Untersuchung der Milz auf sonstige vorhandenen Enzyme hätte über die Grenzen unserer Arbeit hinausgeführt.

Wir lassen die ausführlichen Versuchsserien folgen:

\section{Versuchsserie I.}

Einem kräftigen Foxbastard wird õ Stunden nach einer gehörigen Mahlzeit ( ${ }^{1 / 2}$ Pfund Blutwurst + Weissbrot) die Milz exstirpiert. Das Organ zeigt keine hyperämische Schwellung. Es wiegt 12,ō $\mathrm{g}$.

Die Milz wird nach den Angaben Luciani's mit Kieselgur im Mörser zerrieben, das fünffache Volumen $4 \%$ iger Borsäure hinzugesetzt und das Infus 6 Stunden bei $38^{\circ} \mathrm{C}$. im Brutschrank stehen gelassen. Darauf wurde es filtriert.

Mit dem Infus wurden folgende Versuche angestellt:

1. $20 \mathrm{ccm}$ des Filtrats werden mit gleicher Menge verdünnter Salzsäure in ein Kölbchen gebracht. Wir weichen an dieser Stelle insofern von der Versuchsanordnung Lucianis' ab, als wir zur Ansäuerung des Infuses eine Salzsäure von geringerer Azidität benutzten. $\mathrm{Zu}$ dieser ersten Versuchsserie benutzten wir statt $4 \%$ ige $0,4 \%$ ige Salzsäure.

Als Kontrollprobe dient ein Gemisch von $20 \mathrm{ccm}$ Borsäure und $20 \mathrm{ccm}$ $0,4 \%$ ige Salzsäure.

In jede der beiden Flüssigkeiten wird eine zwischen Filtrierpapier ausgepresste Fibrinflocke von $0,5 \mathrm{~g}$ Gewicht getan, die vorher 30 Minuten in einer $0,2 \%$ gen Salzsäure gelegen hatte, und dann die beiden Kölbchen auf 2 Stunden in den Brutschrank gestellt. Temperatur $38^{\circ} \mathrm{C}$. Nach 2 Stunden wird der Inhalt der beiden Kölbchen abfiltriert. Die Fibrinflocke aus dem zweiten Kölbchen (Borsäure + Salzsäure) war stark gequollen, voluminös. Das Fibrin im ersten Kölbchen (Milzinfus + Salzsäure) hat dagegen an Volumen eingebüsst.

Vor und nach dem Aufenthalt im Brutschrank sind je $2 \times 5 \mathrm{ccm}$ Filtrat zur Stickstoffbestimmung nach Kjeldahl abgenommen worden.

Es waren vorhanden in $5 \mathrm{ccm}$ :

vor dem Aufenthalt im Brutschrank . . . 0,0016 g N, nach dem Aufenthalt im Brutschrank. . . . 0,0016 g N,

also in beiden Fällen eine gleiche Stickstoffmenge. Damit ist festgestellt, dass kein verdauender Einfluss des Milzinfuses auf das Blutfibrin bestanden hat.

2. $5 \mathrm{ccm}$ des Infuses werden mit $0,4 \%$ iger Salzsäure zu gleichen Teilen zersetzt und eine Verdauungsprobe mit Mett'schen Röhrchen angesetzt. Es zeigt sich auch nach 36 Stunden keine Spur von Verdauung.

3. Das wie im vorhergehenden Versuche angesäuerte Infus wird nach der Methode Gross auf Verdauungsfähigkeit geprüft. Die Probe fällt vollständig negativ aus.

\section{Versuchsserie II.}

Zum Versuche dient ein alter fetter Dachsbastard. 5 Stunden vor der Milzexstirpation erhält er eine reichliche Mahlzeit, bestehend aus 1/2 Pfund rohem 
Milz und Magenverdauung und der angebliche Pepsingehalt der Milz. 613

Fleisch, Weissbrot und Zucker mit Wasser angerübrt. Der Hund wird durch einen Hammerschlag getötet. Bei der Sektion finden sich im Magen noch Nahrungsüberreste, die Lymphgefässe des Darmes sind prall gefüllt, die Milz hyperämisch. Das Organ wird in derselben Weise wie in Versuch I behandelt, nur mit dem Unterschied, dass wir uns ganz an die Anordnung Lu ciani's halten und eine 4\% \% ige Salzsäure anwenden.

Mit dem Infus werden folgende Versuche angestellt:

1. In derselben Weise wie in der Versuchsserie I wurden zwei Kölbchen angesetzt. Kölbchen I enthält $20 \mathrm{ccm}$ Milzinfus $+20 \mathrm{ccm} 4 \%$ ige Salzsäure,

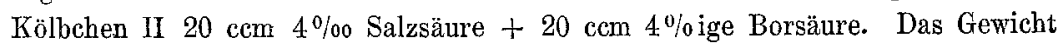
des Fibrins ( $2 \mathrm{~g}$ ) wird bei vollständiger Trockenheit bestimmt. Der Aufenthalt im Brutschrank dauert 3 Stunden. Vorher und nachher werden je $2 \times 5 \mathrm{ccm}$ von I zur Stickstoffbestimmung abgenommen. Diese ergibt, dass in $5 \mathrm{ccm}$ gelöst sind: vor dem Aufenthalt im Brutschrank . . . . 0,0025 g N, nach dem Aufenthalt im Brutschrank. . . . 0,0047 g N.

Es war somit der Stickstoffgehalt in $5 \mathrm{ccm}$ um $0,0022 \mathrm{~g}$ gewachsen.

2. Eine Verdauungsprobe des angesäuerten Milzinfuses mit Mett'schen Röhrchen zeigte nach 30 Stunden keinen Verdauungserfolg.

3. Fin Verdaungsversuch mit der Methode Gross fiel vollständig negativ aus.

\section{Versuchsserie III.}

Der Hund (Bastard vom deutschen Schäferhund) war seit dem Abend vorher nüchtern. Operation $3 \mathrm{Uhr}$ nachmittag in Morphiumäthernarkose. Die exstirpierte Milz ist sehr gross und hyperämisch. Gewicht $46,5 \mathrm{~g}$. Die Milz wird in derselben Weise wie in den vorigen Versuchen behandelt. Eine kleine Abweichung findet statt. Das trockene Fibrin $(0,5 \mathrm{~g})$ wird in $30 \mathrm{ccm} 2 \%$ iger Salzsäure zum

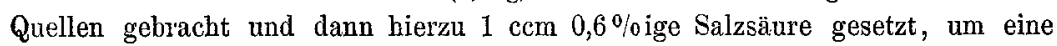
Flüssigkeit von $4 \%$ igem Salzsäuregehalt zu bekommen. Dann werden $31 \mathrm{ccm}$ Milzinfus hinzugetan. Das Kontrollgläschen enthält wieder $4 \%$ ige Borsäure und $4 \%$ ige Salzsäure zu gleichen Teilen. Vor und nach dem zwölfstündigen Aufenthalt im Brutschrank wurden aus jedem der beiden Gläschen je $2 \times 5 \mathrm{ccm}$ zur Stickstoffbestimmung abgenommen. Nach den zwölf Stunden hat sich das Milzinfus getrübt. Die Trockensubstanz des Fibrins im Milzinfus hat um $0,05 \mathrm{~g}$ abgenommen.

Die Trockensubstanzbestimmung der Kontrollproben war nicht möglich, da diese eine schleimig gequollene Masse bildete, die sich rom Filter nicht quantitativ abheben liess.

Die Stickstoff bestimmungen ergeben:

1. für das Milzinfus:

es sind in $5 \mathrm{ccm}$ enthalten vor dem Aufenthalt im Brutschrank . 0,0053 g N, es sind in $5 \mathrm{ccm}$ enthalten nach dem Aufenthalt im Brutschrank. 0,0068 $\mathrm{g} \mathrm{N}$.

Das bedeutet einen Zuwachs von $0,0015 \mathrm{~g} \mathrm{~N}$ in $5 \mathrm{ccm}$.

2. für die Kontrollprobe:

es sind in $5 \mathrm{ccm}$ enthalten vor dem Aufenthalt im Brutschrank. -

es sind in $5 \mathrm{ccm}$ enthalten nach dem Aufenthalt im Brutschrank . 0,0042 g N. 
Die Kontrollfüssigkeit, bestehend aus Borsäure und Salzsäure, hat also in $5 \mathrm{ccm} 0,0027 \mathrm{~g}$ mehr Stickstoff gelöst als Milzinfus + Salzsäure.

\section{Versuchsserie IV.}

Es werden vier Kölbchen angesetzt, enthaltend:

I $40 \mathrm{ccm} 4 \%$ ige Salzsäure,

II $40 \mathrm{ccm} 2 \%$ ige Salzsäure,

III $40 \mathrm{ccm} 0,25 \%$ ige Essigsäure,

IV $40 \mathrm{ccm} 0,125 \%$ ige Essigsäure.

In jedes Kölbchen werden $2 \mathrm{~g}$ gemahlenes trockenes Fibrin getan. Nach 24 stündigem Aufenthalt im Brutschrank sind in den Gläschen an Stickstoff gelöst:

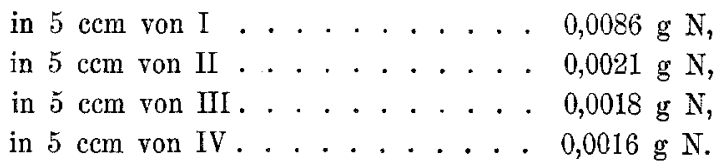

Wir haben im Widerspruch zu den eingangs angeführten Beobachtungen nur in einem Falle bei unseren Versuchshunden 5 Stunden nach reichlicher Mablzeit eine hyperämische Anschwellung der Milz gesehen. In den beiden anderen Fällen verhielt sich die Milz durchaus abweichend. Im Versuch I fanden wir sie nicht hyperämisch, trotzdem der Hund 5 Stunden vorher reichlich gefüttert worden war, und in Versuch III war sie hyperämisch, trotzdem der Hund seit dem Abend zuvor nichts zu fressen bekommen hatte.

Die Beobachtungen Luciani's haben wir vollständig bestätigen können. Wir fanden:

1. dass Blutfibrin in wenig angesäuertem Milzinfus an Volumen verliert.

Wir haben ausserdem sogar konstatieren können:

1. dass bei stärkerer Ansäuerung eine Gewichtsabnahme des Blutfibrins zu konstatieren ist;

2. dass sich im Filtrat eine Zunahme des Stickstoffes findet.

Trotzdem bestreiten wir, dass es sich bei dem Befunde um eine Pepsinwirkung des Milzinfuses handelt. Wir sahen nämlich:

1. die in I. beobachtete Volumenverminderung des Fibrins fand statt ohne Vermehrung des gelösten Stickstoffs. Eine Verdauung hatte also nicht stattgefunden, vielmehr war nur die durch Salzsäure erfolgte Quellung des Fibrins nach Zusatz von Milzinfus weniger stark geworden;

2. Salzsäure + Borsäure lösten mehr Fibrin als Salzsäure + Milzinfus. 
Es ist also die von Lucian $\mathrm{i}$ beobachtete Volumenverminderung der Fibrinsäule - die wir auch konstatiert haben - fälschlich als auf einer peptischen Verdauung beruhend angesehen worden, während es sich in Wirklichkeit nur um eine Schrumpfung der Fibrinsäule handelt. Die von Martin G. Fischer in seinem Buch „Das Ödem ${ }^{1}$ )" mitgeteilten Befunde über die Quellung des Fibrins stehen im Einklang mit unserer Beobachtung.

Fischer fand, dass die kolloiden Stoffe wie das Fibrin bei Gegenwart von Säure, besonders Salzsäure und danach Essigsäure eine starke Affinität zum Wasser haben und bedeutend mehr quellen als in reinem Wasser. Nach Zusatz von Salzen in bestimmer Menge und Konzentration kommt diese Quellung zum Stillstand, oder geht sogar zurück. Es müssen nun in der $\mathrm{Milz}$ quellungshemmende Stoffe vorhanden sein, auf die es zurückzufübren ist, dass das vorher in zweipromilliger Salzsäure gequollene Fibrin nach 21/2 stündigem Aufenthalt in Milzinfus + Salzsäure an Volumen verliert.

Martin G. Fischer machte auch die Beobachtung, dass Sätren, die durch ein Filter von gemahlenem Fibrin liefen, eiweisshaltig wurden, "weil Fibrin in Säurelösung (pseudo)löslich ist". Das Albumen vermindert sich, oder verschwindet gänzlich, wenn genügend Salz der Säurelösung zugesetzt wird.

Es liegt also näher, dass der Stickstoff, den wir in den vorliegenden Versuchen im Filtrat nachgewiesen haben, durch Einwirkung der Salzsäure gelöst wurde. Die stärkere Löslichkeit in Salzsäure allein wie in angesäuertem Milchinfus führt uns zu der Annahme, dass das Milzinfus nicht nur ein quellungshemmendes, sondern auch ein lösungshemmendes Agens enthält.

Aus Versuch IV geht in Übereinstimmung mit den Resultaten von Martin G. Fischer hervor, dass auch Essigsäure in geringerem Maasse als Salzsäure Fibrin zu lösen vermag. Vielleicht ist es möglich, dass auch bei den Versuchen von Hedin und Rowland, die ja verdünnte $(0,25 \%)$ Essigsäure zur Ansäuerung des Milzinfuses benutzten, die Säure eine bedeutsame Rolle spielt. Sollte sich aber in der Tat im Milzinfus ein proteolytisches Enzym einwandsfrei nachweisen lassen, so wäre das für unsere Frage belanglos, als es sich keinesfalls ım Pepsin handeln könnte, weil dieses auch in der Versuchsanordnung von Luciani zum Ausdruck kommen müsste.

1) Martin G. Fischer, Das Öden. Dresden 1910. 
Ja selbst, wenn in der Milz Pepsin gefunden würde, könnten wir der Theorie nicht zustimmen, die dasselbe zum Pepsingehalt des Magens in irgendeine Beziehung setzt, da die Versuche des ersten Teiles gezeigt haben, dass die Exstirpation der Milz auf die Verdaunngstätigkeit des Magens keinen Einfluss hat.

Das Ergebnis der Arbeit ist also:

1. Die Exstirpation der Milzhat keinenverringernden Einfluss auf die Verdaungstätigkeit des Magens.

2. Nach Milzexstirpation tritt eine Lymphocytose auf, es besteht aber kein Parallelismus $z$ wischen dieser und der Absonderung des Magenpepsins.

3. Das Infus der hyperämisch geschwollenen Milz enthält kein Pepsin oder Pepsinogen.

4. Die stickstofflösende Einwirkung des angesäuerten Milzinfuses auf Blutfibrin ist der auflösenden. Wirkung der zugesetzten Säure zuzuschreiben.

5. Die Volumenverminderung des Fibrins in Milzinfus ist zum grössten Teil auf eine Schrumpfung des Fibrins zurückzuführen. 